\title{
Decreased Eph receptor-A1 expression is related to grade in ovarian serous carcinoma
}

\author{
YUNFENG JIN $^{1}$, YI ZOU ${ }^{1}$, LINLING WAN ${ }^{1}$, MINGMING LU $^{1}$, YA LIU ${ }^{2}$, \\ GUOQIN HUANG ${ }^{3}$, JIANDONG WANG ${ }^{4}$ and QINGHUA XI ${ }^{1}$
}

\begin{abstract}
${ }^{1}$ Department of Obstetrics and Gynecology, Affiliated Hospital of Nantong University, Nantong, Jiangsu 226001;
${ }^{2}$ Department of Obstetrics and Gynecology, Affiliated Haian People's Hospital of Nantong University, Nantong, Jiangsu 226600; ${ }^{3}$ Department of Obstetrics and Gynecology, Affiliated Maternal and Child Health Care Hospital of

Nantong University, Nantong, Jiangsu 226018; ${ }^{4}$ Department of Pathology, Jinling Hospital,

Nanjing University School of Medicine, Nanjing, Jiangsu 210002, P.R. China
\end{abstract}

Received June 28, 2017; Accepted October 9, 2017

DOI: $10.3892 / \mathrm{mmr} .2018 .8528$

\begin{abstract}
Eph receptor-A1 (EphA1) was the first member of the erythropoietin producing hepatocellular carcinoma (Eph) family of receptor tyrosine kinases. Although the roles of EphA1 in the tumorigenesis of various human cancers have been investigated, few studies have focused on ovarian carcinoma. The present study aimed to explore the profile of EphA1 expression in ovarian carcinomas, to analyzed the association between EphA1 expression and clinicopathologic parameters, and to investigate the roles of overexpressed EphA1 in ovarian cancer cells. EphA1 protein was detected in ovarian cancer cell lines and in a set of formalin-fixed tissues, including normal fallopian tube, ovarian benign serous cystadenoma, borderline serous tumors and serous carcinoma. Ovarian cancer cell lines HO8910 and A2780 were transiently transfected with EphA1-pCMV6-GFP plasmid, and the proliferation and apoptosis of cells were measured. The association between EphA1 expression and clinicopathological parameters was statistically analyzed. EphA1 expression was negative in HO8910 and weakly positive in A2780 cells. The proliferation rate was significantly reduced in ovarian cancer cells after transfection with EphA1 plasmid compared with cells transfected with mock plasmid or untreated cells, but no obvious alteration in apoptosis was detected among these groups. EphA1 expression was positively detected in all normal fallopian tubes $(10 / 10$,
\end{abstract}

Correspondence to: Dr Qinghua Xi, Department of Obstetrics and Gynecology, Affiliated Hospital of Nantong University, 20 Xisi Road, Nantong, Jiangsu 226001, P.R. China

E-mail: ntdxfsyyxqh@163.com

Dr Jiandong Wang, Department of Pathology, Jinling Hospital, Nanjing University School of Medicine, 305 Zhong San Dong Lu, Nanjing, Jiangsu 210002, P.R. China

E-mail: jd_wang@outlook.com

Key words: Eph receptor-A1, grade, ovarian serous carcinoma
$100 \%)$ and ovarian benign serous cystadenomas (12/12, 100\%) as well as in some borderline serous tumors $(9 / 15,60 \%)$ and ovarian serous carcinomas (33/76, 43.42\%). EphA1 expression was associated with grade of ovarian serous carcinomas, with loss of EphA1 more often observed in high-grade tumors $(\mathrm{P}=0.016)$ and high Ki67 index tumors $(\mathrm{P}=0.007)$. These data suggest that EphA1 might be a useful marker for distinguishing low grade from high-grade ovarian serous carcinoma.

\section{Introduction}

Epithelial ovarian cancer (EOC) is the most common type of ovarian cancer, accounting for over $90 \%$ of ovarian cancers, and is one of the three most common cancers in females $(1,2)$. EOC is associated with high morbidity and mortality rates owing to the typical late stage of the disease at diagnosis; up to $75 \%$ of females with EOC are diagnosed at advanced stages because there are few symptoms in the early stage (3). Ovarian serous carcinoma is the most common histological type of ovarian cancer, accounting for $70-80 \%$ of all newly diagnosed patients, and the most common and most aggressive subtype of EOC (4). Over the past 30 years, advances in surgery and chemotherapy have had little impact on overall patient survival, and current treatment leads to relapse in the majority of patients. This situation calls for investigation of the pathogenesis of ovarian serous carcinoma and identification of molecular markers for early diagnosis and treatment.

Erythropoietin producing hepatocellular carcinoma (Eph) receptors constitute the largest subfamily of receptor tyrosine kinases that bind membrane-bound ligands called ephrins (5). The Eph/ephrin interactions emanate their signals in a bidirectional manner into adjacent cells, followed by internalization and degradation of the complexes (6). Eph/ephrin signaling is proposed to participate in a wide spectrum of developmental processes through its capacity to regulate cellular adhesion, migration, or repulsion and tissue/cell boundary formation (7-11). Beyond their initial role in developmental processes, Ephs and ephrins are also involved in a broad range of processes directly related to tumor progression and metastasis $(6,12-15)$. 
Eph receptor-A1 (EphA1), the first member of the Eph receptor tyrosine kinase family to be discovered, was isolated as a gene that was amplified in a carcinoma cell line and shown to be located on chromosome 7q34 (16). EphrinA1 is the highest affinity binding ligand for EphA1, although EphA1 also binds ephrin $\mathrm{A} 3$ and $\mathrm{A} 4$ with lower affinity. Whole-mount in situ hybridization showed overlapping expression of EphA1, ephrinA1, and ephrinA3 in the streak and the posterior paraxial mesoderm during early mouse development (17). Activation of EphA1 can inhibit cell spreading and migration in a Rho-ROCK-dependent manner (18). These results suggested that interaction of EphA1 and ephrinA1/A3 plays a role in tumor development. EphA1 expression has been detected in several types of human cancer. EphA1 mRNA and protein were detected in human epidermis at a high level, but EphA1 protein expression was reduced in non-melanoma skin cancers derived from the epidermis (19). In a previous study, we explored EphA1 expression in colorectal cancer, gastric cancer, and renal carcinoma and analyzed the correlation between EphA1 expression and clinicopathological parameters (20-22). Our data suggest that EphA1 is expressed in human cancers at highly varying levels. Expression of EphA1 protein has not yet been determined in ovarian serous carcinoma. The purpose of this study is to investigate the expression of EphA1 protein in ovarian serous adenocarcinoma and its association with clinical parameters.

\section{Materials and methods}

Cell lines and tissue samples. Human ovarian cancer cell lines HO8910 and A2780 used in the present study were purchased from the cell resource center of the Shanghai Institute of life Sciences, Chinese Academy of Sciences. HO8910 and A2780 were maintained in RPMI 1640 medium (Invitrogen, Carlsbad, CA, USA) supplemented with $10 \%$ fetal bovine serum (Gibco; Invitrogen), $100 \mathrm{U} / \mathrm{ml}$ penicillin, and $100 \mathrm{mg} / \mathrm{ml}$ streptomycin in a $5 \% \mathrm{CO}_{2}$ and $95 \%$ atmosphere at $37^{\circ} \mathrm{C}$ with cell culture plates (Thermo Fisher Scientific, Inc., Waltham, MA, USA).

Clinical specimens were collected from the Department of Pathology of Affiliated Hospital of Nantong University from January 2001 to January 2013. Samples consisted of 10 normal fallopian tubes (age, 24-50 years; average, 43.1), 12 ovarian benign serous cystadenoma tumor tissues (age, 23-62 years; average, 38.5), 15 borderline serous tumors (age, 22-51 years; average, 34.5 ) and 76 ovarian serous carcinoma tissues (age, 27-69 years; average, 48.7). Generally, matched normal (or non-tumor) and tumor tissue from same patient are subjected to detection when we investigate a gene expression profile in certain cancer. However, serous ovarian carcinoma is very special. First of all, according to recent research results, serous ovarian carcinoma is not derived from ovarian epithelial cells, but from fallopian tube (23). Secondly, there usually almost no normal ovarian surface epithelial was available in ovarian tumor tissues. Formalin-fixed and paraffin-embedded tumor tissues were sectioned at 4- $\mu \mathrm{m}$ thickness. Each tumor was classified according to WHO Classification Tumors of Female Reproduction Organs (23). Data were acquired with approval from the Ethics Committee of the Affiliated Hospital of Nantong University.
EphA1 plasmid transfection. The plasmid EphA1-pCMV6GFP eukaryotic expression vector kit was purchased from OriGene Technologies, Inc (Rockville, MD, USA). HO8910 and A2780 cells were each divided into three groups: EphA1 transfected group (EphA1-TG), mock group (MG), and untransfected group (UTG). The MG group and the EphA1-TG group were transiently transfected with plasmid pCMV6-GFP and plasmid EphA1-pCMV6-GFP respectively using Lipofectamine 2000 according to the manufacturer's instructions. The UTG group did not receive any treatment. The transfection rate for EphA1-pCMV6-GFP and mock was checked by observation of GFP with a fluorescence microscope and by RT-PCR amplification of EphA1 mRNA. The protocol for amplification of EphA1 mRNA was the same as in our previous report (20). For EphA1, the sense primer is 5'-ATC TTTGGGCTGCTGCTTGG-3' and the antisense primer is 5'-GCTTGTCCTCTCGATCCACATC-3'. For housekeeping gene GAPDH, the sense primer is 5'-CCAGGTGGTCTCCTC TGACTT-3' and the antisense primer is 5'-GTTGCTGTAGCC AAATTCGTTGT-3'.

Determination of cell viability (MTT assay). HO8910 and A2780 cells were seeded in 96-well flat-bottomed plates with 5,000 cells per well in $100 \mu \mathrm{l}$ of complete RPMI 1640 medium, followed by incubation at $37^{\circ} \mathrm{C}\left(5 \% \mathrm{CO}_{2}\right.$ and $95 \%$ air $)$ for $24 \mathrm{~h}$ to allow the cells to reach $70 \%$ confluence. The cells were transiently transfected and cultured for $48 \mathrm{~h}$. The supernatant was carefully removed, and $100 \mu \mathrm{l}$ medium and $20 \mu \mathrm{l}$ of a $5 \mathrm{mg} / \mathrm{ml}$ MTT solution (Thermo Fisher Scientific, Inc.) were added to each well and incubated for $4 \mathrm{~h}$ at $37^{\circ} \mathrm{C}$. The excess MTT was then aspirated. Viable cells internalize the MTT into their mitochondria. The formazan crystals formed in cells were dissolved by addition of $150 \mu 1$ of dimethyl sulfoxide (DMSO). After shaking for $1 \mathrm{~h}$, the absorbance was measured at $540 \mathrm{~nm}$ in a multiwall scanning spectrophotometer.

Apoptosis. Apoptosis of cells after transfection for $72 \mathrm{~h}$ with EphA1-pCMV6-GFP and mock plasmid was detected using an Annexin V-FITC apoptosis detection kit (Qiagen GmbH, Hilden, Germany). In brief, cells were collected after digestion with $0.25 \%$ trypsin and rinsed. The cells were resuspended in binding buffer with $5 \mu 1$ Annexin V-FITC and $5 \mu 1$ propidium iodide (PI). After incubation at room temperature for $10 \mathrm{~min}$ in the dark, Annexin V-FITC/PI binding was measured by flow cytometry (excitation, $488 \mathrm{~nm}$; emission, $530 \mathrm{~nm}$ ) using the phycoerythrin emission signal detector (FL1 for detection of FITC, and FL2 for detection of PI).

Immunocytochemical (ICC) and immunohistochemical (IHC) staining. ICC and IHC staining was performed by the Envision method. For ICC staining, cells were grown on glass coverslips to $70 \%$ confluence, washed with PBS, and fixed with cold $75 \%$ ethanol for $10 \mathrm{~min}$ on ice. The cells were incubated in 3\% $\mathrm{H}_{2} \mathrm{O}_{2}$ for 10 min and then at $4^{\circ} \mathrm{C}$ overnight with an anti-EphA1 polyclonal antibody (AO1047a, ABGENT) at a 1:100 dilution in Antibody Diluent (Zymed; Invitrogen). After a wash with PBS, the cells were incubated with secondary antibody (Dako, Ely, UK) for $20 \mathrm{~min}$ at room temperature. Color development was performed with 3,3-diaminobenzidine (DAB). Nuclei were lightly counterstained with hematoxylin. 

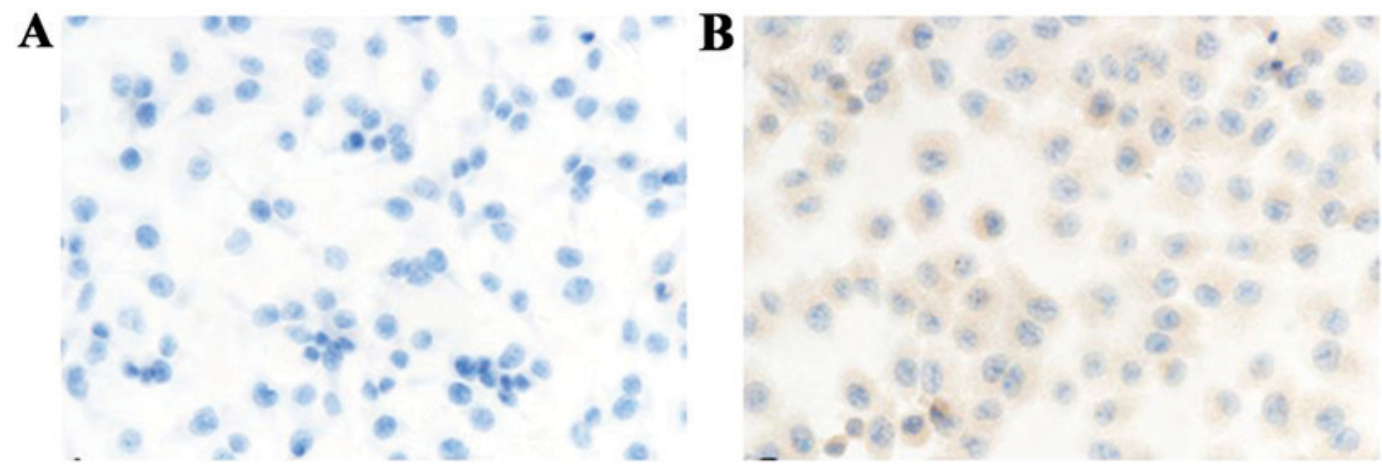

Figure 1. EphA1 expression was detected in ovarian cancer cell lines by immunocytological staining. (A) Expression of EphA1 protein was negative in the human ovarian cancer cell line HO8910. (B) Expression of EphA1 protein was weakly positive in the human ovarian cancer cell line A2780 (original magnification, x400). Eph1A1, Eph receptor-A1.

For IHC staining, 4- $\mu \mathrm{m}$ thick sections were deparaffinized in xylene. After rehydration through a graded ethanol series, the sections were autoclaved in $10 \mathrm{mM}$ citrate buffer ( $\mathrm{pH}$ 6.0) at $120^{\circ} \mathrm{C}$ for $2 \mathrm{~min}$ for antigen retrieval and then cooled to $30^{\circ} \mathrm{C}$ and washed with PBS ( $\mathrm{pH}$ 7.3). After non-specific sites had been blocked with $3 \% \mathrm{H}_{2} \mathrm{O}_{2}$ for 10 min, the sections were incubated at $4^{\circ} \mathrm{C}$ overnight with an anti-EphA1 polyclonal antibody and washed with PBS. The subsequent steps were the same as for ICC. Two pathologists independently assessed the immunostained slides, and any differences in the staining scores were resolved by consensus.

IHC scoring and quantification. Cytoplasmic staining was considered positive staining. The scoring for percentage of immunoreactive tumor cells was as follows: $0,0 \% ; 1,<20 \%$; $2,20-50 \%$; and $3,>50 \%$. The staining intensity was scored and stratified as follows: 0 , negative; 1 , weak; 2 , moderate; and 3 , strong. A final immunoreactivity score (IRS) was obtained for each of the cases by multiplying the percentage score and the intensity score. Protein expression levels were further analyzed by classifying IRS values as negative (IRS value $<4$ ) or positive (IRS value $\geq 4$ ) (24).

Statistical analysis. Cell experiments were repeated three times and data were expressed as mean \pm standard deviation (mean \pm standard deviation). Results were analyzed by one-way ANOVA. The $\chi^{2}$ test (Fisher's exact test) was used to assess the associations of EphA1 protein expression with clinicopathological variables. Two-sided P-values $<0.05$ were considered statistically significant. All analyses were performed by SPSS software (version 16.0; SPSS, Inc., Chicago, IL, USA).

\section{Results}

Expression of EphA1 in ovarian cancer cell lines. EphA1 expression in human ovarian cancer cell lines HO8901 and A2780 was examined by immunocytochemistry. EphA1 staining was located in the cytoplasm. The expression of EphA1 protein was negative in HO8910 cells and weakly positive in A2780 cells (Fig. 1).

Transfection of EphAl gene. The ovarian cancer cells were observed using a fluorescence microscope after transient transfection with pCMV6-GFP or EphA1-pCMV6-GFP plasmids. Green fluorescence was observed in ovarian cancer cells transfected with EphA1-TG and MG, but not in UTG (Fig. 2A). EphA1 mRNA expression in cells of the EphA1-TG group was detected by RT-PCR (127 bp), with GAPDH mRNA as an internal control (416 bp) (Fig. 2B).

Proliferation of HO8910 and A2780 cell lines after EphAI transfection. The MTT assay was performed to determine the proliferative effect of HO8910 and A2780 ovarian cancer cells transfected with EphA1 and the data were analyzed using two-sample independent t-test. The proliferation rate of both HO8910-EphA1-TG and A2780-EphA1-TG cells was significantly reduced compared with that in mock and untransfected groups (Fig. 3).

Apoptosis in HO8910 and A2780 cell lines after EphAl transfection. Apoptosis was measured in HO8910 and A2780 ovarian cancer cells using flow cytometry. There was no significant difference in apoptosis among the EphA1 transfected group, mock, and untransfected groups for both HO8910 and A2780 cells (Fig. 4).

EphAl expression in normal fallopian tube and ovarian serous tumors. EphA1 staining in normal fallopian tube, ovarian benign serous cystadenoma, borderline serous tumors and serous carcinoma was located predominantly in the cytoplasm with diffuse positive expression (Fig. 5). Positive EphA1 staining was detected in all normal fallopian tubes $(10 / 10)$ and ovarian benign serous cystadenomas (12/12). EphA1 protein was positively detected in some samples of borderline serous tumors (9/15) and ovarian serous carcinoma (33/76) (Table I).

EphAl expression correlated with clinicopathological features. The relationship between EphA1 expression and clinicopathological parameters was shown in Table II. Statistical analysis of the association between EphA1 expression and clinicopathological features revealed a significant relationship between EphA1 expression and tumor grade $(\mathrm{P}=0.016)$ and Ki67 $(\mathrm{P}=0.007)$. No significant association of EphA1 expression and other features was found in this study. 
A

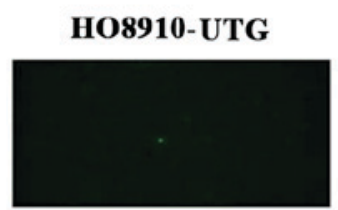

HO8910-MG

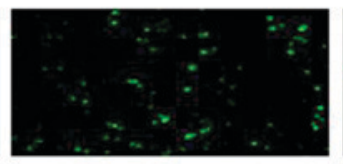

A2780-MG
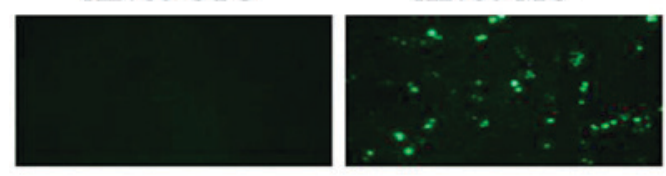

HO8910-EphA1-TG

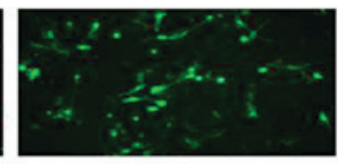

A2780-EphA1-TG

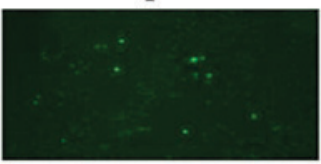

B

400 bp
200 bp
100 bp
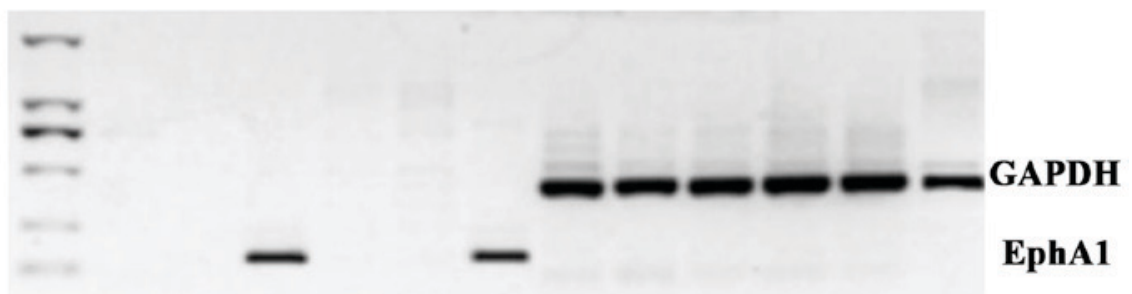

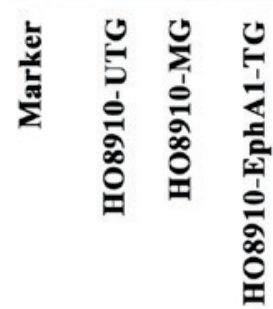

苞

勿

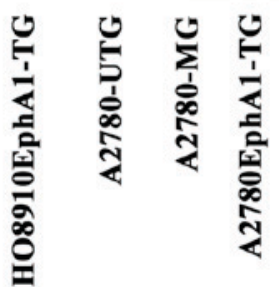

Figure 2. Transfection of EphA1-pCMV6-GFP and mock plasmid in ovarian cancer cell lines. (A) GFP signal was not observed in untransfected groups (HO8910-UTG and A2780-UTG); GFP signal was observed in mock groups (HO8910-MG and A2780-MG) and transfected groups (HO8910-EphA1-TG and A2780-EphA1-TG). (B) Transfection of EphA1 in ovarian cancer cell lines was confirmed by RT-PCR. EphA1 mRNA was amplified in transfected groups (HO8910-EphA1-TG and A2780-EphA1-TG), was not found in untransfected groups (HO8910-UTG and A2780-UTG) and mock groups (HO8910-MG and A2780-MG). Eph1A1, Eph receptor-A1; EphA1-TG, EphA1 transfected group; MG, mock group; UTG, untransfected group.

Table I. EphA1 expression in normal fallopian tubes, ovarian benign serous cystadenomas, and serous carcinomas.

\begin{tabular}{lcccc}
\hline & & \multicolumn{2}{c}{ EphA1 } & Positive \\
\cline { 3 - 4 } Group & No. & Negative & Positive & $(\%)$ \\
\hline $\begin{array}{l}\text { Normal fallopian } \\
\text { tube }\end{array}$ & 10 & 0 & 10 & 100.00 \\
$\begin{array}{l}\text { Serous cystadenoma } \\
\text { Borderline serous }\end{array}$ & 12 & 0 & 12 & 100.00 \\
$\begin{array}{l}\text { tumors } \\
\text { Serous carcinoma }\end{array}$ & 76 & 6 & 9 & 60.00 \\
\hline
\end{tabular}

Eph1A1, Eph receptor-A1.

\section{Discussion}

Roles of the receptor tyrosine kinases in both normal physiology and oncogenesis have been well established. The genes that encode Eph receptors, the largest subfamily of receptor tyrosine kinases, are primarily considered to be classic oncogenes. Overexpression of EphA1 has been reported in several human cancers (25-27); however, reduced expression of EphA1 also has been detected in prostate cancer cell lines (28), basal

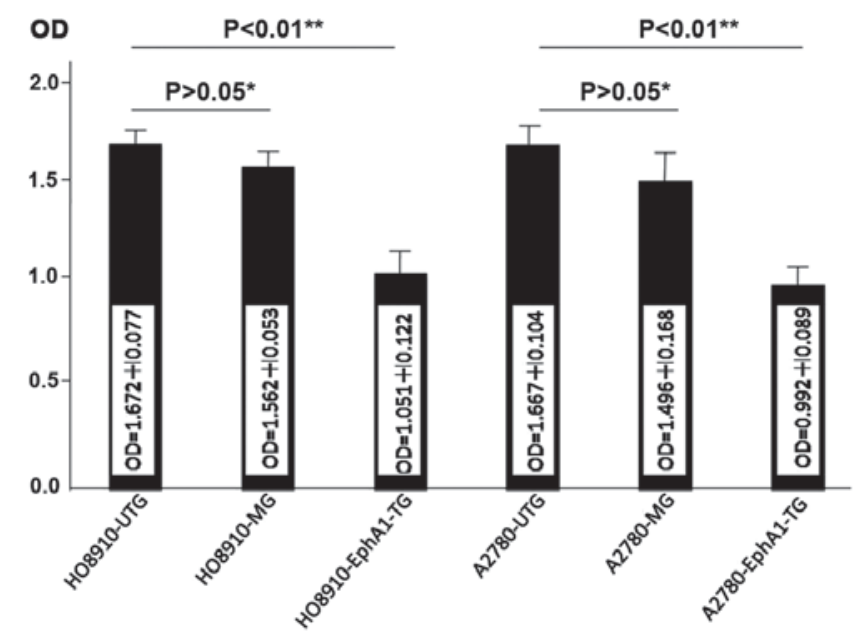

Figure. 3 The MTT assay was performed to determine the proliferative effect of HO8910 and A2780 ovarian cancer cells transfected with EphA1. No significant difference was observed in UTG and MG groups of both HO8910 and A2780 $(\mathrm{P}>0.05)$. The proliferation rate in transfected groups (HO8910-EphA1-TG and A2780-EphA1-TG) was significantly reduced compared with $\mathrm{MG}(\mathrm{P}<0.01)$ and UTG $(\mathrm{P}<0.01)$. Eph1A1, Eph receptor-A1; EphA1-TG, EphA1 transfected group; MG, mock group; UTG, untransfected group.

cell carcinomas and squamous cell carcinomas of the skin (19), and colorectal cancer (20). Therefore, whether EphA1 is an oncogene has been questioned. We previously reported that 
A
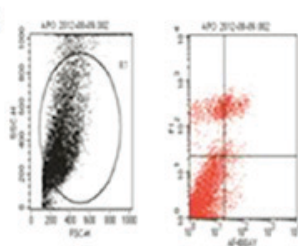

HO8910-UTG

E
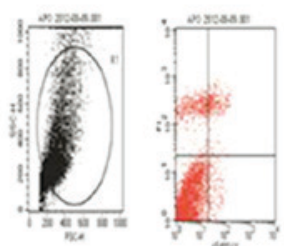

A2780-UTG
B

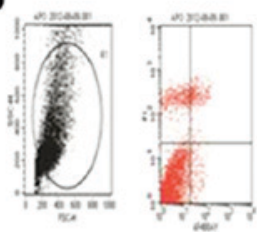

H08910-MG
C

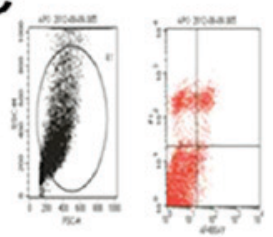

HO8910-EphA1-TG
D

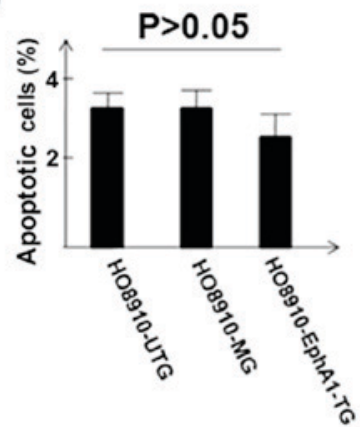

F
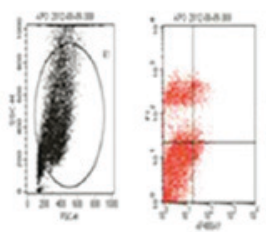

$\mathbf{G}$

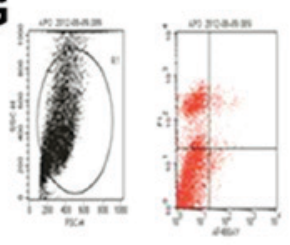

A2780-EphA1-TG
$\mathrm{H}$

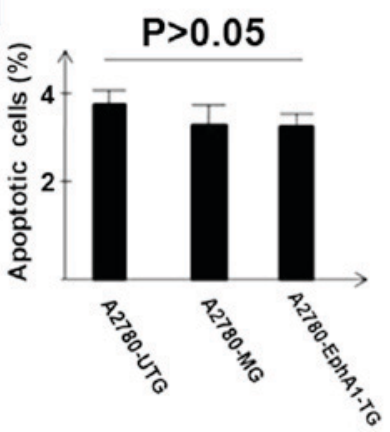

Figure 4. Apoptosis was measured in HO8910 and A2780 ovarian cancer cells using flow cytometry. There was no significant difference in apoptosis among the UTG (A and E), MG (B and F), and EphA1-TG (C and G) in both HO8910 (D; P>0.05) and A2780 cells (H; P>0.05). Eph1A1, Eph receptor-A1; EphA1-TG, EphA1 transfected group; MG, mock group; UTG, untransfected group.

A

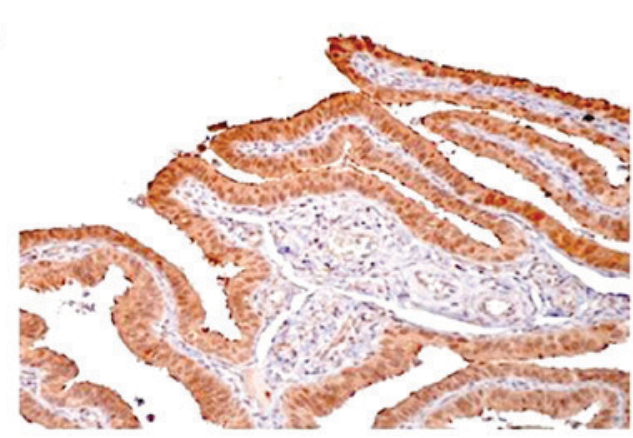

C

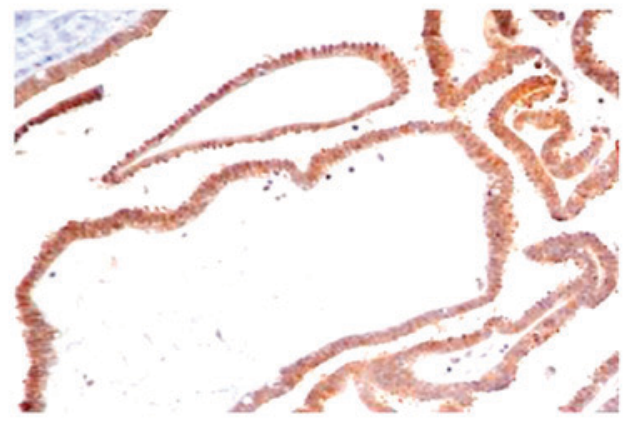

B
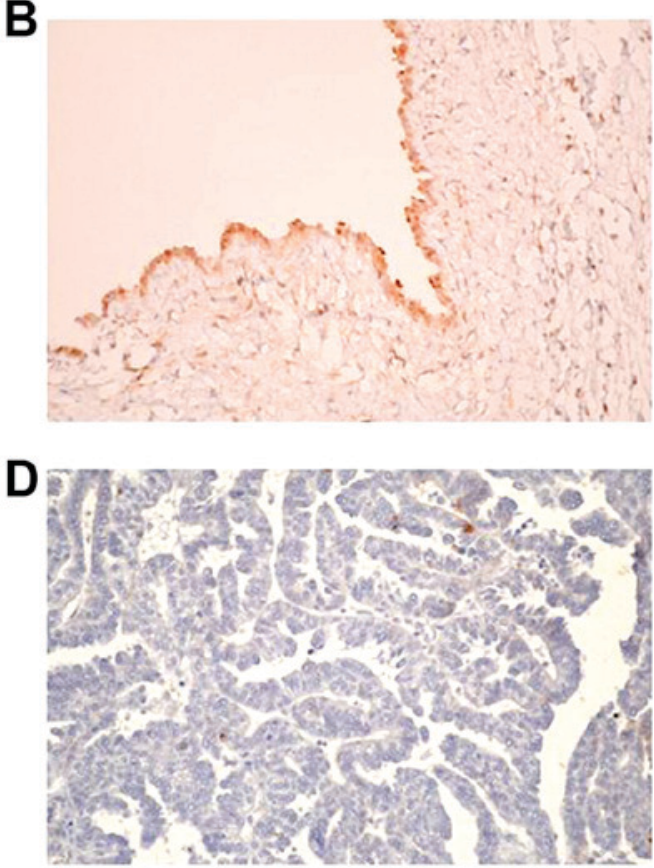

Figure 5. EphA1 expression was detected in ovarian tissues by immunohistochemistry. EphA1 expression was positive in normal fallopian tube (A), benign serous cystadenoma (B), and low-grade ovarian serous carcinoma (C) and negative in high-grade ovarian serous carcinoma (D). Original magnification, $\mathrm{x} 400$. Eph1A1, Eph receptor-A1.

EphA1 expression was associated with metastasis in esophageal squamous cell carcinoma (29), Gleason score in prostate cancer (30), invasion and metastasis in colorectal cancer (20), and metastasis in gastric cancer (21). Comprehensive studies show obvious differences in EphA1 expression among different tissues and different tumor types.
In this study, EphAl expression was negative in HO8910 and weakly positive in A2780 ovarian cancer cells. In addition, loss of EphA1 expression was found in most ovarian serous carcinoma tissues compared with normal fallopian tube and benign tumor. Our data suggest that EphAl is downregulated in ovarian serous carcinoma. In contrast, Wong et al reported 
Table II. Relationship between EphA1 expression and clinicopathologic parameters in ovarian serous carcinomas.

\begin{tabular}{|c|c|c|c|c|c|c|}
\hline \multirow[b]{2}{*}{ Parameters } & \multirow[b]{2}{*}{ No. } & \multicolumn{2}{|c|}{ EphA1 } & \multirow[b]{2}{*}{ Positive \% } & \multirow[b]{2}{*}{$\chi^{2}$} & \multirow[b]{2}{*}{ P-value } \\
\hline & & - & + & & & \\
\hline \multicolumn{7}{|c|}{ Clinical stages } \\
\hline I+II & 15 & 6 & 9 & 60.00 & & \\
\hline III+ IV & 61 & 37 & 24 & 39.34 & 2.091 & 0.148 \\
\hline \multicolumn{7}{|l|}{ Grade } \\
\hline Low & 12 & 3 & 9 & 75.00 & & \\
\hline High & 64 & 40 & 24 & 37.50 & 5.784 & 0.016 \\
\hline \multicolumn{7}{|l|}{ Metastasis } \\
\hline Yes & 30 & 19 & 11 & 36.67 & & \\
\hline No & 46 & 24 & 22 & 47.83 & 0.920 & 0.337 \\
\hline \multicolumn{7}{|l|}{$\begin{array}{l}\text { Position of } \\
\text { tumors }\end{array}$} \\
\hline Single & 32 & 16 & 16 & 50.00 & & \\
\hline Double & 44 & 27 & 17 & 38.64 & 0.974 & 0.324 \\
\hline \multicolumn{7}{|c|}{$\begin{array}{l}\text { Maximum } \\
\text { diameter }(\mathrm{cm})\end{array}$} \\
\hline$\leq 5$ & 29 & 13 & 16 & 55.17 & & \\
\hline $5-10$ & 21 & 15 & 6 & 28.57 & & \\
\hline$\geq 10$ & 17 & 11 & 6 & 35.29 & 3.938 & 0.140 \\
\hline No data & 9 & 4 & 5 & & & \\
\hline \multicolumn{7}{|l|}{ Age (years) } \\
\hline$\leq 50$ & 30 & 17 & 13 & 43.33 & & \\
\hline $50-55$ & 16 & 12 & 4 & 25.00 & & \\
\hline$\geq 55$ & 30 & 14 & 16 & 53.33 & 3.410 & 0.182 \\
\hline \multicolumn{7}{|l|}{ Ki67 } \\
\hline$>20 \%$ & 52 & 35 & 17 & 68.42 & & \\
\hline$\leq 20 \%$ & 24 & 8 & 16 & 31.58 & 7.715 & 0.007 \\
\hline
\end{tabular}

Eph1A1, Eph receptor-A1.

that EphA1 mRNA was upregulated in EOC with positive immunostaining of ephrin receptor A1 (31) and Herath et al reported that overexpression of EphA1 mRNA strongly correlated with the high-affinity ligand ephrin A1 in advanced ovarian cancer (32). We interpret the difference in results between these papers and ours as follows: First, the experimental samples used by Herath et al (32) and Wong et al (31) included ovarian serous, mucinous, endometrioid, and clear cell cancer, whereas we focused on ovarian serous cancer. Second, mechanisms regulating EphA1 protein level may be important in ovarian cancer and EphA1 mRNA expression may show an inconsistent trend. The possible mechanisms need to be further explored. We previously proved that hypermethylation of a $\mathrm{CpG}$ island in the EphA1 promoter region leads to downregulation of EphA1 in colorectal cancer (20). We therefore deduced that methylation of DNA might be one of the mechanisms for reduced expression of EphA1 in ovarian serous cancers. Other possible regulatory mechanisms include EphA1 mutation, microRNA, deacetylation, and gene deletion. We plan to intensively investigate these molecular mechanisms in future studies.

Ki67 is a marker of proliferation expressed exclusively during active phases of the cell cycle. It is commonly assessed by IHC in clinical settings to judge cell proliferative activity. It has been correlated with clinical outcome and is considered to be an indicator of prognosis. Interestingly, our data show that loss of EphA1 was more often observed in high Ki67 index tumors $(\mathrm{P}=0.007)$. On the other hand, the MTT proliferation assay showed that overexpression of EhpA1 gene inhibited the proliferation of HO8910 and A2780 tumor cells, this is consistent with what observed in tumor tissues. Overexpression of EphA1 in ovarian cancer cell lines did not affect cell apoptosis. Our results suggest that EphA1 may play a role in ovarian cancer as a tumor suppressor but is not a key suppressor gene in ovarian tumorigenesis.

Histologic grade has been shown to be an important prognostic factor in cases of ovarian serous carcinoma. Although the ovarian grading system has evolved over the years, there is no universally accepted classification. The Federation of Gynecology and Obstetrics (FIGO) grading system typically analyzes architectural pattern, nuclear/cytologic atypia, mitotic index, or a combination of these features. Molecular pathological research has contributed to improved knowledge of the different subtypes of ovarian cancer. The World Health Organization Classification System of Ovarian Cancer, published in 2014 by Kurman et al (23), eliminated the older practice of grading serous tumors on a continuum (grade 1,2, or 3) and instead differentiates low-grade serous and high-grade serous ovarian cancers as two distinct diseases. It is now widely accepted that low-grade and high-grade serous tumors are essentially distinct diseases exhibiting distinct genetic alterations, molecular patterns, and clinical behaviors. Low-grade serous carcinoma develops from well-recognized precursors and behaves in an indolent fashion. It is characterized by specific mutations including KRAS, BRAF, and ERBB2 and is relatively genetically stable (1). In contrast, high-grade serous ovarian carcinoma is characterized by advanced stage at diagnosis, frequent TP53 mutation, rapid progression, and high responsiveness to platinum-based chemotherapy (33). Although high-grade and low-grade serous carcinomas are usually easily distinguished, it may be difficult to discriminate between them in some carcinomas and can especially challenging in small tissue samples (34). This is the first study demonstrating that EphA1 protein is significantly correlated with tumor grade in ovarian serous carcinoma, with negative expression of EphA1 more often found in ovarian high-grade serous cancers $(\mathrm{P}=0.016)$. Our data suggest that EphA1 may be a new molecular marker for grading ovarian serous carcinoma.

In conclusion, EphA1 expression is decreased in ovarian serous carcinoma compared with normal fallopian tube and benign ovarian serous cystadenoma. Decreased EphA1 expression was more often detected in high-grade tumors. Our data suggest that EphA1 may be a new marker for grading and prognosis in ovarian serous adenocarcinoma.

\section{Acknowledgements}

The authors wish to thank the patients who participated in this study. This study was supported by grants from the National 
Natural Science Foundation of China (grant no. 81371611) and the National Basic Research Priorities Program 973 Project (grant no. 2014CB744504) from the Ministry of Science and Technology of China.

\section{References}

1. Ciucci A, Zannoni GF, Buttarelli M, Martinelli E, Mascilini F, Petrillo M, Ferrandina G, Scambia G and Gallo D: Ovarian low and high grade serous carcinomas: Hidden divergent features in the tumor microenvironment. Oncotarget 7: 68033-68043, 2016.

2. Siegel RL, Miller KD and Jemal A: Cancer statistics, 2016. CA Cancer J Clin 66: 7-30, 2016.

3. Seebacher V, Reinthaller A, Koelbl H, Concin N, Nehoda R and Polterauer S: The impact of the duration of adjuvant chemotherapy on survival in patients with epithelial ovarian cancer-a retrospective study. PLoS One 12: e0169272, 2017.

4. Takekuma M, Wong KK and Coleman RL: A long-term surviving patient with recurrent low-grade serous ovarian carcinoma treated with the MEK1/2 inhibitor, selumetinib. Gynecol Oncol Res Pract 3: 5, 2016.

5. Unified nomenclature for Eph family receptors and their ligands, the ephrins. Eph Nomenclature Committee. Cell 90: 403-404, 1997.

6. Pasquale EB: Eph receptors and ephrins in cancer: Bidirectional signalling and beyond. Nat Rev Cancer 10: 165-180, 2010.

7. Drescher U: The Eph family in the patterning of neural development. Curr Biol 7: R799-R807, 1997.

8. Mellitzer G, Xu Q and Wilkinson DG: Eph receptors and ephrins restrict cell intermingling and communication. Nature 400 77-81, 1999.

9. Xu Q, Mellitzer G, Robinson V and Wilkinson DG: In vivo cell sorting in complementary segmental domains mediated by Eph receptors and ephrins. Nature 399: 267-271, 1999.

10. Holmberg J, Clarke DL and Frisén J: Regulation of repulsion versus adhesion by different splice forms of an Eph receptor. Nature 408: 203-206, 2000

11. Cooke J, Moens C, Roth L, Durbin L, Shiomi K, Brennan C, Kimmel C, Wilson S and Holder N: Eph signalling functions downstream of Val to regulate cell sorting and boundary formation in the caudal hindbrain. Development 128: 571-580, 2001.

12. Herath NI and Boyd AW: The role of Eph receptors and ephrin ligands in colorectal cancer. Int J Cancer 126: 2003-2011, 2010.

13. Takano H, Nakamura T, Tsuchikawa T, Kushibiki T, Hontani K, Inoko $\mathrm{K}$, Takahashi $\mathrm{M}$, Sato $\mathrm{S}$, Abe $\mathrm{H}$, Takeuchi $\mathrm{S}$, et al: Inhibition of Eph receptor A4 by 2,5-dimethylpyrrolyl benzoic acid suppresses human pancreatic cancer growing orthotopically in nude mice. Oncotarget 6: 41063-41076, 2015.

14. Husa AM, Magić Ž,Larsson M,Fornander T and Pérez-Tenorio G: EPH/ephrin profile and EPHB2 expression predicts patient survival in breast cancer. Oncotarget 7: 21362-21380, 2016.

15. Mateo-Lozano S, Bazzocco S, Rodrigues P, Mazzolini R, Andretta E, Dopeso H, Fernández Y, Del Llano E, Bilic J, Suárez-López L, et al: Loss of the EPH receptor B6 contributes to colorectal cancer metastasis. Sci Rep 7: 43702, 2017.

16. Hirai H, Maru Y, Hagiwara K, Nishida J and Takaku F: A novel putative tyrosine kinase receptor encoded by the eph gene. Science 238: 1717-1720, 1987.

17. Duffy SL, Steiner KA, Tam PP and Boyd AW: Expression analysis of the Ephal receptor tyrosine kinase and its high-affinity ligands Efna1 and Efna3 during early mouse development. Gene Expr Patterns 6: 719-723, 2006.

18. Yamazaki T, Masuda J, Omori T, Usui R, Akiyama $H$ and Maru Y: EphA1 interacts with integrin-linked kinase and regulates cell morphology and motility. J Cell Sci 122: 243-255, 2009
19. Hafner C, Becker B, Landthaler $M$ and Vogt T: Expression profile of Eph receptors and ephrin ligands in human skin and downregulation of EphA1 in nonmelanoma skin cancer. Mod Pathol 19: 1369-1377, 2006

20. Dong Y, Wang J, Sheng Z, Li G, Ma H, Wang X, Zhang R, Lu G, $\mathrm{Hu} \mathrm{Q}$, Sugimura H and Zhou X: Downregulation of EphA1 in colorectal carcinomas correlates with invasion and metastasis. Mod Pathol 22: 151-160, 2009.

21. Wang J, Dong Y, Wang X, Ma H, Sheng Z, Li G, Lu G, Sugimura $\mathrm{H}$ and Zhou X: Expression of EphA1 in gastric carcinomas is associated with metastasis and survival. Oncol Rep 24 $1577-1584,2010$

22. Wang X, Liu Y, Cao G, Zhang X, Xu H, Xu H and Wang J: Expression of the EphA1 protein is associated with Fuhrman nuclear grade in clear cell renal cell carcinomas. Int J Clin Exp Pathol 8: 6821-6827, 2015.

23. Kurman RJ, Carcangiu ML, Herrington CS, and Young RH: WOH Classification of Tumours of Female Reproductive Organs. International Agency for Research on Cancer, Lyon, 2014.

24. Miyazaki K, Inokuchi M, Takagi Y, Kato K, Kojima K and Sugihara K: EphA4 is a prognostic factor in gastric cancer. BMC Clin Pathol 13: 19, 2013.

25. Fox BP and Kandpal RP: Invasiveness of breast carcinoma cells and transcript profile: Eph receptors and ephrin ligands as molecular markers of potential diagnostic and prognostic application. Biochem Biophys Res Commun 318: 882-892, 2004.

26. Nakagawa M, Inokuchi M, Takagi Y, Kato K, Sugita H, Otsuki S, Kojima K, Uetake H and Sugihara K: Erythropoietin-producing hepatocellular A1 is an independent prognostic factor for gastric cancer. Ann Surg Oncol 22: 2329-2335, 2015.

27. Giaginis C, Tsoukalas N, Bournakis E, Alexandrou P, Kavantzas N, Patsouris E and Theocharis S: Ephrin (Eph) receptor A1, A4, A5 and A7 expression in human non-small cell lung carcinoma: Associations with clinicopathological parameters, tumor proliferative capacity and patients' survival. BMC Clin Pathol 14: 8, 2014.

28. Fox BP, Tabone CJ and Kandpal RP: Potential clinical relevance of Eph receptors and ephrin ligands expressed in prostate carcinoma cell lines. Biochem Biophys Res Commun 342: 1263-1272, 2006.

29. Wang J, Ma J, Dong Y, Shen Z, Ma H, Wang X, Shi S, Wu J, Lu G, Peng L and Zhoud X: High expression of EphA1 in esophageal squamous cell carcinoma is associated with lymph node metastasis and advanced disease. APMIS 121: 30-37, 2013.

30. Peng L, Wang H, Dong Y, Ma J, Wen J, Wu J, Wang X, Zhou X and Wang $\mathrm{J}$ : Increased expression of EphA1 protein in prostate cancers correlates with high Gleason score. Int J Clin Exp Pathol 6: 1854-1860, 2013.

31. Wong YL, Dali AZ, Mohamed Rose I, Jamal R and Mokhtar NM: Potential molecular signatures in epithelial ovarian cancer by genome wide expression profiling. Asia Pac J Clin Oncol 12: e259-e268, 2016.

32. Herath NI, Spanevello MD, Sabesan S, Newton T, Cummings M, Duffy S, Lincoln D, Boyle G, Parsons PG and Boyd AW: Over-expression of Eph and ephrin genes in advanced ovarian cancer: Ephrin gene expression correlates with shortened survival. BMC cancer 6: 144, 2006.

33. Mignogna C, Staropoli N, Botta C, De Marco C, Rizzuto A, Morelli M, Di Cello A, Franco R, Camastra C, Presta I, et al: Aurora kinase a expression predicts platinum-resistance and adverse outcome in high-grade serous ovarian carcinoma patients. J Ovarian Res 9: 31, 2016.

34. Gu Y, Li F, Qian N, Chen X, Wang H and Wang J: Expression of EphB6 in ovarian serous carcinoma is associated with grade, TNM stage and survival. J Clin Pathol 69: 448-453, 2016 